International Journal of Bifurcation and Chaos, Vol. 22, No. 7 (2012) 1250161 (8 pages)

(C) World Scientific Publishing Company

DOI: $10.1142 / \mathrm{S} 0218127412501611$

\title{
PACEMAKERS IN A CAYLEY TREE OF KURAMOTO OSCILLATORS
}

\author{
PABLO M. GLEISER \\ Centro Atómico Bariloche, Instituto Balseiro, \\ 8400 Río Negro, Argentina \\ LUCE PRIGNANO, CONRAD J. PÉREZ-VICENTE \\ and ALBERT DÍAZ-GUILERA \\ Departament de Física Fonamental, \\ Universitat de Barcelona 08028 Barcelona, Spain
}

Received December 3, 2010; Revised March 11, 2011

\begin{abstract}
In this work, we study a system of Kuramoto oscillators with identical frequencies in a Cayley tree. Heterogeneity in the frequency distribution is introduced in the root of the tree, allowing for analytical calculations of the phase evolution. In this work, we study a system of Kuramoto oscillators with identical frequencies in a Cayley tree. Heterogeneity in the frequency distribution is introduced in the root of the tree, allowing for analytical calculations of the phase evolution. This simple case can be regarded as a starting point in order to understand how to extract topological features of the connectivity pattern from the dynamic state of the system, and vice versa, for the general situation of a set of phase oscillators located on a tree-like network.
\end{abstract}

Keywords: Synchronization; Cayley tree; Kuramoto model.

\section{Introduction}

The heterogeneous structure of complex networks plays a fundamental role in the dynamical properties of systems of interacting units, a paradigmatic example being synchronization, conceived as an emerging coherent behavior [Pikovsky et al., 2001; Osipov et al., 2007]. Understanding the role of connectivity in synchronization has been the subject of intense research in recent years [Arenas et al., 2008]. These studies have been carried out along two main directions. The first is the search for necessary and sufficient conditions granted that a population of units obeying simple dynamical rules is able to synchronize, in the framework of dynamical systems theory [Winfree, 1980]. The second is the definition and in-depth investigation of precise models of phase oscillators, one of the best known being a model proposed by Kuramoto [Kuramoto, 1975; Acebrón et al., 2005] where the interaction between the units follows a sine function.
Our work goes along this second line, since we consider a system of coupled Kuramoto oscillators. While in his original work Kuramoto, as well as many other authors later, considered oscillators equally coupled to each other, we take into account a heterogeneous interaction pattern. Here our choice is that of a very simple topology, the Cayley tree, for which we are able to compute analytically some interesting quantities.

In the large majority of previous studies, the natural frequencies of the oscillators are taken from a given distribution. The nonzero width of this distribution allows the units to follow different trajectories, while, on the other hand, the interaction term leads to the units approaching their phases. It was shown that there exists a critical value of the interaction strength above which the system gets into the coherent regime. The precise value at which this transition takes place depends on the width of the frequency distribution, but not on the details 
as far as it is bounded. If all the natural frequencies of the oscillators are identical, then the system will end up in a synchronized (same phases) state, in general, for any connected topology and any initial conditions (with just some limitations [DiazGuilera \& Arenas, 2008]). In our case, for simplicity, we choose a particular bimodal frequency distribution where only one single oscillator has a natural frequency different from that of the rest of the population. Then, we can say that this case can be regarded as the simplest perturbation to the trivial homogeneous case.

Up to now, the role of a particular oscillator, characterized by a higher natural frequency, has been analyzed in detail for several kinds of regular as well as for random networks, and analytical calculations have been performed. In works like [Kori \& Mikhailov, 2004] this special oscillator is not affected by the others, while in [Radicchi \& Meyer-Ortmanns, 2006] the interaction between a particular element and its neighbors is triggered by a parameter and can vary from totally asymmetrical to symmetrical. Anyway, in both cases it is usual to refer to the oscillator playing this special role as the pacemaker.

In our particular case, we locate the pacemaker at the root of the Cayley tree focusing on the dynamical consequence of the perturbation introduced by its presence. In this way we are able to obtain analytic results for the phase evolution of the oscillators.

\section{The Model}

We consider as the network structure of our system a Cayley tree of variable radii and coordination numbers (Fig. 1).

The nodes follow the dynamics described by the Kuramoto model of phase oscillators [Kuramoto, 1984; Acebrón et al., 2005]. All of them have the same natural frequency (taken to be zero without loss of generality) except for the one located at the root of the tree, that has a different natural frequency $\omega$. We will refer to this node as the pacemaker. The equations are

$$
\begin{aligned}
& \dot{\phi}_{p}=\omega+\sum_{j} a_{p j} \sin \left(\phi_{j}-\phi_{p}\right) ; \\
& \dot{\phi}_{i}=\sum_{j} a_{i j} \sin \left(\phi_{j}-\phi_{i}\right) \quad \forall i \neq p,
\end{aligned}
$$

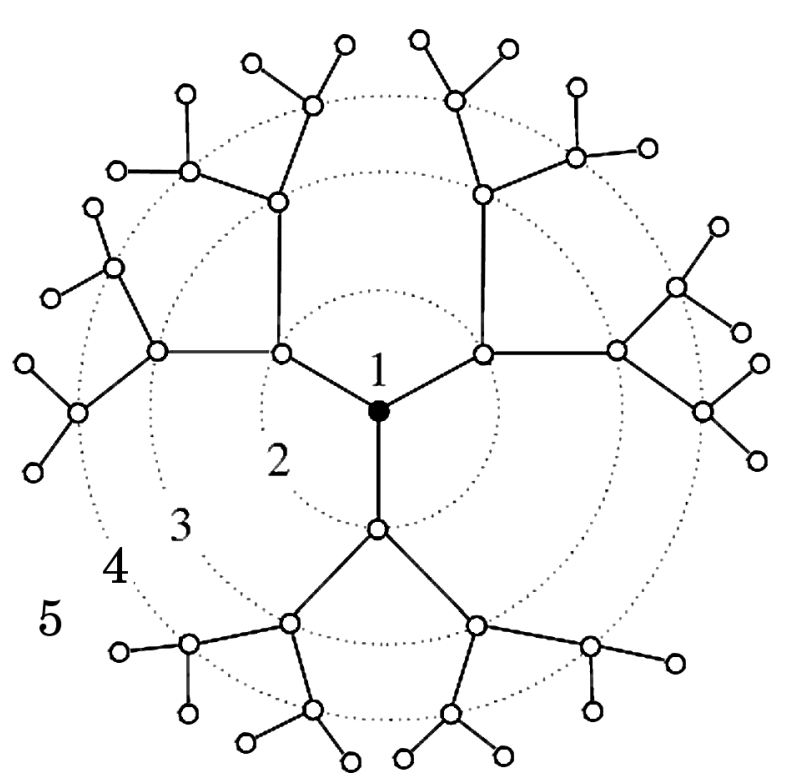

Fig. 1. Cayley tree with coordination number $q=3$ and radius $R=5$. The pacemaker is located at the root of the tree (filled circle).

where the first one is for the pacemaker, the second for all the rest. The matrix $a_{i j}$ is the corresponding adjacency matrix for the network.

Notice that for our natural frequencies distribution it is never possible to reach synchronization in an equal phase state, that is, a state where all the interaction terms vanish. It is also important to stress synchronization does not depend on the choice we made of a null natural frequency for all the $N-1$ oscillators except the pacemaker. Indeed, we can change the mean value of the natural frequencies distribution by just rotating the frame, without any modification of dynamical properties of the system. For instance, we could choose a zero mean distribution, that can be obtained applying the following transformation to the phases: $\varphi_{i} \rightarrow$ $\varphi_{i}+\Omega t$, in which $\Omega=\omega / N$ is the first moment of the distribution.

\section{Characterizing the Global Behavior}

The transition from an incoherent to a synchronized state is usually described by means of an order parameter introduced by Kuramoto:

$$
z(t)=\frac{1}{N} \sum_{j} \exp \left(i \phi_{j}\right) .
$$

The amplitude of this quantity is proportional to $1 / N$ in the incoherent regime whereas it grows 


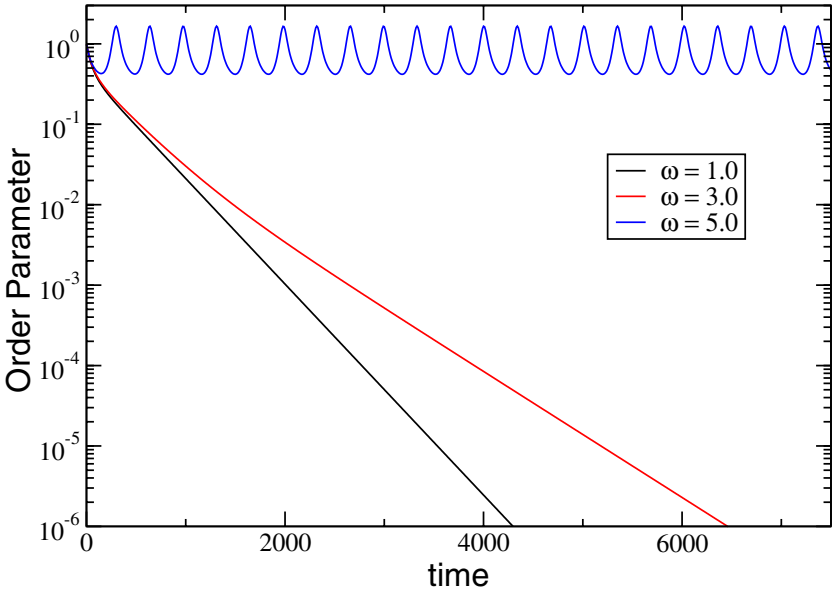

(a)

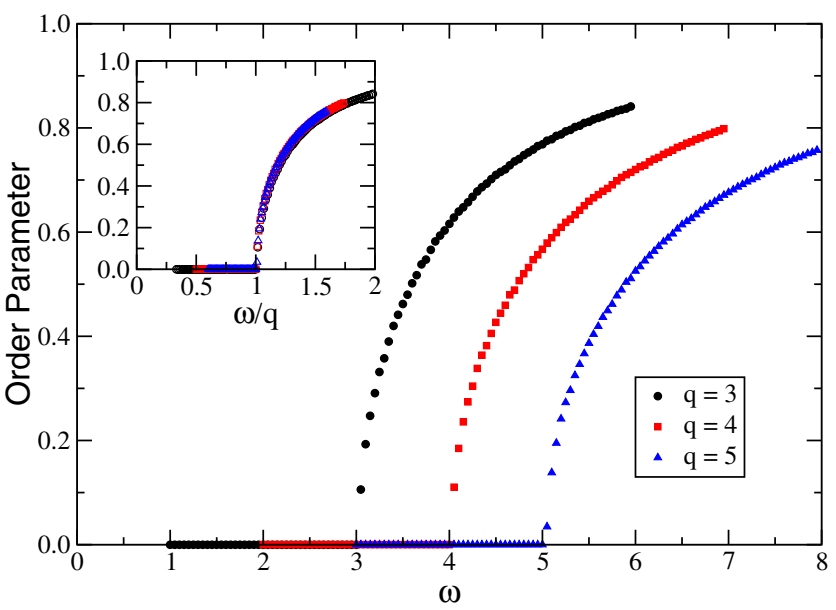

(b)

Fig. 2. (a) Effective frequency dispersion order parameter $\Delta_{\omega}$ (4) versus time. (b) Normalized frequency dispersion order parameter $r_{\omega}(5)$ versus natural frequency of the pacemaker $\omega$.

with decreasing $\omega$ in the phase-locked state and asymptotically it tends to 1 as $\omega$ tends to 0 (phase-locking state). Anyway, since our system can undergo a phase synchronization only at local level, this parameter is not an appropriate one in order to describe its global dynamical behavior.

Then, following [Prignano \& Diaz-Guilera, 2012], we analyze an order parameter already introduced and used in [Buzna et al., 2009] and [SendiñaNadal et al., 2008] that measures the effective frequency dispersion:

$$
\Delta_{\omega}=\sqrt{\frac{1}{N} \sum_{i \in N}\left[\dot{\phi}_{i}-\langle\omega\rangle\right]^{2}} .
$$

In Fig. 2(a) we present the time evolution of this order parameter for a Cayley tree with a coordination number $q=3$, and radius $R=5$, with a total of $N=94$ nodes. The results correspond to initial conditions with all the phases equal to zero. For frequencies below a critical value $\omega_{c}$ the order parameter decays exponentially to zero, revealing that for long times there is no dispersion in the effective frequencies and the system becomes synchronized. Above $\omega_{c}$ the system does not reach equilibrium, and the order parameter presents an oscillating behavior.

Note also that the frequency dispersion presented in Eq. (4) is not normalized. We divide the frequency dispersion by its maximum allowed value $\frac{\omega}{N} \sqrt{N-1}$, and thus obtain a normalized order parameter:

$$
r_{\omega}=\sqrt{\frac{1}{N-1} \sum_{i \in N}\left[\frac{\dot{\phi}_{i}}{\langle\omega\rangle}-1\right]^{2}} .
$$

In Fig. 2(b) we present the stationary value of the normalized order parameter as a function of the pacemaker frequency $\omega$. As noted, for values above $\omega_{c}$ the system is not in equilibrium, however, it is possible to define a mean value around which the order parameter oscillates. The figure shows the behavior observed for three different values of the coordination number, $q=3,4$ and 5. As $q$ grows, the critical value also grows accordingly. The inset in the figure shows the data collapse obtained by scaling the natural frequency of the pacemaker with the coordination number, $\omega / q$.

\section{Results}

In order to analyze the relation between the topology of the connectivity pattern and the ability of the system to reach a synchronized state, we notice that when we increase the regulator frequency $\omega$ then there will be some oscillators that cannot keep the phase difference, breaking the synchronized state. The left-hand side of Eq. (2) is indeed bounded because of the sine terms, whereas the right term increases as the regulator frequency is increased. The same happens in Eq. (1). Consequently, there will be a transition from a synchronized to an incoherent state, at a precise value of $\omega$. Thus, we can define the natural frequency of the pacemaker 
critical value as follows:

$$
\frac{\omega_{p}^{c}}{\omega}>\omega_{p}^{c} \nexists \text { frequency locking state }
$$

i.e. $\omega_{c}$ is that value of the frequency of the regulator above which no synchronized state exists. In the case of a Cayley tree topology, it is possible to analytically calculate this quantity. We start noticing that, since the existence of a steady state is a necessary condition for a frequency-locked state, we can compute the constant value that all the effective frequencies are going to take for a stationary condition. First of all, we notice, following [Prignano \& Diaz-Guilera, 2012], that summing up all the Eqs. (1) and (2) we have:

$$
\sum_{i=1}^{N} \Omega_{i}=\omega
$$

where $\Omega_{i}$ are the stationary effective frequencies. Consequently, if they all take the same value, this will be $\omega / N$. In this case, the system is in a synchronized state where all the frequencies are equal and constant. Now we can calculate, as a function of the radius $R$ and of the connectivity number $q$, that is the maximum value $\omega$ such that all the oscillators reach an effective frequency equal to $\omega / N$. Since the left side of the phase evolution equation for the pacemaker (1) is bounded, it is clear that a bound exists. Indeed, it is when all the sine terms in that expression take values -1 that we have the maximum $\omega$ able to satisfy this equation. So we have found the following condition for the critical frequency:

$$
\omega_{c} \leq q \frac{N}{N-1}=q+\left[\sum_{r=0}^{R-1}(q-1)\right]^{-1} .
$$

where in the last equality we used the expression $N=1+q \sum_{r=0}^{R-1}(q-1)$.

Locating the pacemaker at the root is a special situation of the more general case discussed in [Prignano \& Diaz-Guilera, 2012]. There it was shown how this particular configuration, where all the neighbors of the pacemakers keep a phase difference equal to $-\pi / 2$, is not always allowed. Indeed, the interplay of other equations, different from that of the pacemaker, may cause a breaking of the stationary condition earlier.

In order to take into account all these contributions, according to [Prignano \& Diaz-Guilera, 2012], for an arbitrary topology of the interactions, we can generalize inequality (7) in the following form:

$$
\omega_{c} \leq N\left[\frac{K_{\text {out }}}{N_{\text {out }}}\right]_{\text {min }}
$$

where $\left[\frac{K_{\text {out }}}{N_{\text {out }}}\right]_{\min }$ is the minimum ratio for a connected component of the connectivity pattern including the pacemaker, $N_{\text {out }}$ being the number of nodes outside the considered group and $K_{\text {out }}$ the number of "external links" connecting the component with the rest of the network. This expression reduces to (7) if we take into account just the pacemaker itself, since in this case we have $N_{\text {out }}=N-1$ and $K_{\text {out }}$ coincides with the degree of the pacemaker, $q$ in our case. Doing so, we are not minimizing the ratio in Eq. (8), we are instead maximizing its denominator but often it is a very good approximation. Moreover, if the connectivity pattern has a tree-like topology, that is, there is no cycle on it, Eq. (9) is not just a bound, but the exact expression of the critical frequency.

The precise case of a Cayley tree topology of the connectivity pattern has been analyzed in [Prignano \& Diaz-Guilera, 2012], where an explicit expression of the inequality (8) is provided for a tree of radius $R$ and coordination number $q$, where the pacemaker is located at distance $r$ from the center:

$$
\omega_{c}^{(r)}=\frac{N}{N-\sum_{i=0}^{R-r}(q-1)^{i}} .
$$

It is easy to show that the new bound simply coincides with (7) when the distance between the pacemaker and the center of the Cayley tree is $r=0$. Consequently, we can rewrite Eq. (7) in the form of an equality:

$$
\omega_{c}=q+\left[\sum_{r=0}^{R-1}(q-1)\right]^{-1},
$$

it is the critical value for the natural frequency of a pacemaker located at the root of a Cayley tree.

In order to better describe the dynamical behavior of the system above the critical point, it is useful to compute the order parameter defined by Kuramoto. Indeed, even if it is not useful in order to identify the transition point, this quantity provides interesting qualitative information about the global time evolution of the phases when the coherent state is broken. 
First, we can ask ourselves what is the behavior of this quantity exactly at the critical point. In order to do this, we can rewrite the order parameter defined in (3) as

$$
z(t)=\frac{1}{N}\left[\exp \left(i \phi_{p}\right)+\sum_{j=1}^{q} \exp \left(i \phi_{j}\right)+\sum_{l=1}^{N-q-1} \exp \left(i \phi_{l}\right)\right],
$$

where the units labeled $j$ are the first neighbors of the pacemaker, and those labeled $l$ all the rest. Now, using the critical condition for the synchronization $\phi_{j}-\phi_{p}=-\frac{\pi}{2}$, we have:

$$
\begin{aligned}
& z(t)=\frac{1}{N}\left[\exp \left(i \phi_{p}\right)+\sum_{j=1}^{q} \exp \left(i\left(\phi_{p}-\frac{\pi}{2}\right)\right)+\sum_{l=1}^{N-q-1} \exp \left(i \phi_{l}\right)\right] \\
& z(t)=\frac{1}{N}\left[\exp \left(i \phi_{p}\right)\left(1+q \exp \left(-i \frac{\pi}{2}\right)\right)+\sum_{l=1}^{N-q-1} \exp \left(i \phi_{l}\right)\right] \\
& z(t)=\frac{1}{N}\left[\exp \left(i \phi_{p}\right)(1-i q)+\sum_{l=1}^{N-q-1} \exp \left(i \phi_{l}\right)\right]
\end{aligned}
$$

In general, this quantity is not computable analytically since the phases are mutually coupled by transcendental equations. However, if we consider a star shaped network, that is, a one level Cayley tree $(R=1)$, then this last equation becomes

$$
z(t)=\frac{1}{N}\left[\exp \left(i \phi_{p}\right)(1-i q)\right]
$$

and we have an analytical expression for the behavior of the Kuramoto order parameter. For this simple case, it is also possible to achieve an analytical expression for the time evolution of effective frequencies:

$$
\dot{\phi}_{i \neq p}=-\sin \left[2 \arctan \left(\left[A \tan \left(-\frac{A}{2} t+B\right)-N\right] \frac{1}{\omega}\right)\right]
$$

and

$$
\dot{\phi}_{p}=\omega+(1-N) \dot{\phi}_{i \neq p},
$$

where $A=\sqrt{\omega^{2}-N^{2}}$ and $B=\arctan \left(\frac{N}{A}\right)$. Plotting the values of the order parameters (14) and (15) in the complex plane, for a sufficiently large time window, we can see what happens below and above the critical point. In the first case, we have a circle whose radius decreases as we increase the natural frequency of the pacemaker. At the critical point this circle takes its minimal size. If we further increase the value of $\omega$ then we get something that is completely different, although the figure is still a closed curve with central symmetry. In Fig. 3 we plot a sequence of images of the values taken by parameter (14) during time windows of increasing duration.

In Fig. 4, we plot the final frame of some sequences analogous to the previous one, for different values of $\omega$, in the case of a star. All these plots are obtained by numerical simulation, except for the critical order parameter for the star in Fig. 4. Both figures show how, above the critical point, these values approach initially a circle smaller than the critical one, then they depart from it rapidly. It implies that the effective frequencies of all oscillators approach each other during a certain time lag, but then they cannot reach a stable constant value. This situation is also confirmed in Fig. 5, where we plot expressions (16) and (17) for several values of $\omega \geq \omega_{c}$. It is easy to notice how all the frequencies reach the same meta-stable value if $\omega$ is not too far from $\omega_{c}$, keeping it during a time lag that decreases on increasing $\omega$, until it disappears. Then the frequencies of the pacemaker and those of the other oscillators get completely apart. In all cases, the frequencies show a periodical trend with a common period.

Finally, we wish to compare this analytically solved simple case (the star) with the general case 

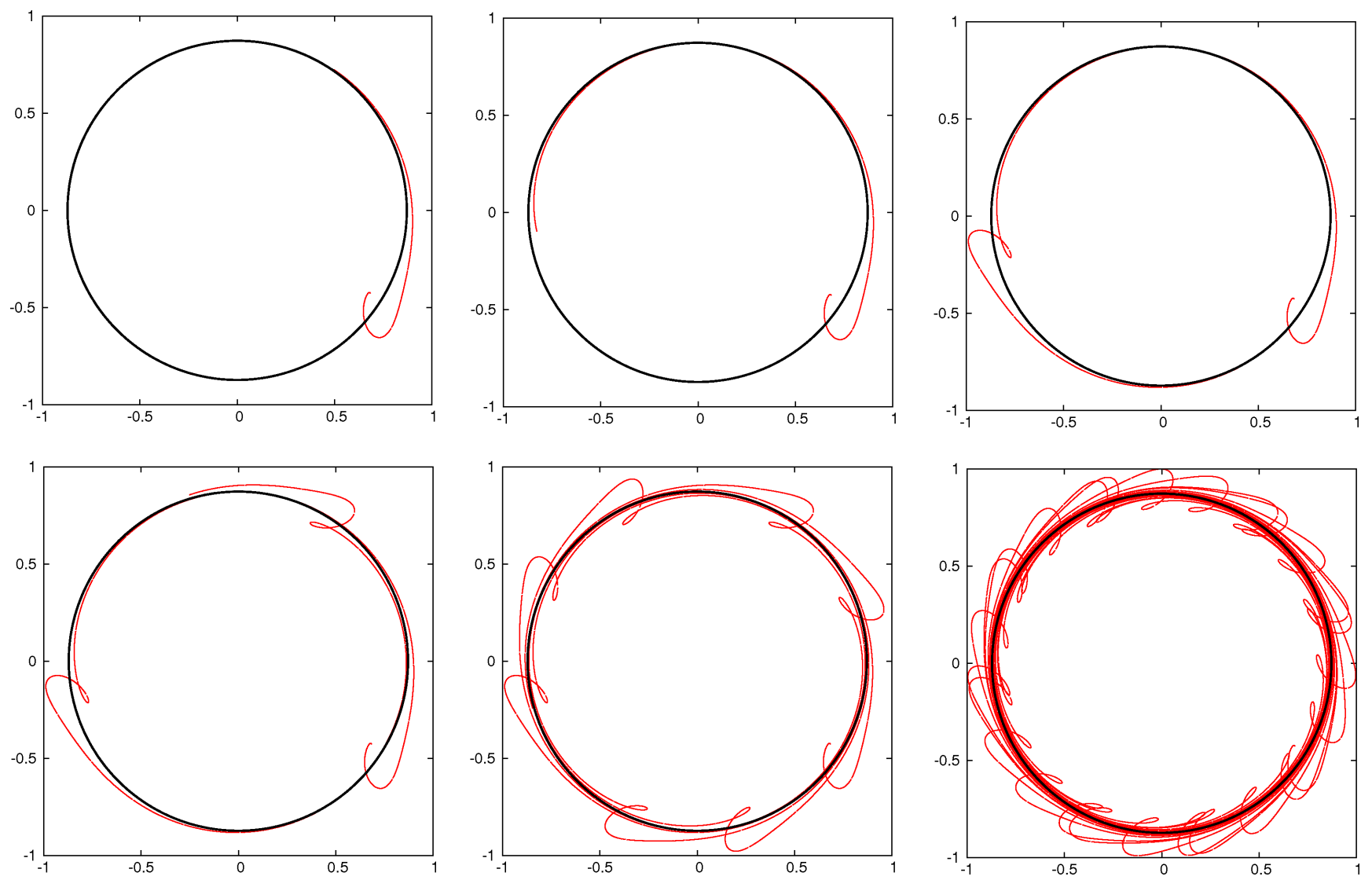

Fig. 3. Values taken in the complex plane by parameter (14) for a Cayley tree of radius $R=4$ and coordination number $q=3$, during time windows of increasing duration. The pacemaker natural frequency is $\omega=1.01 \omega_{c}$.

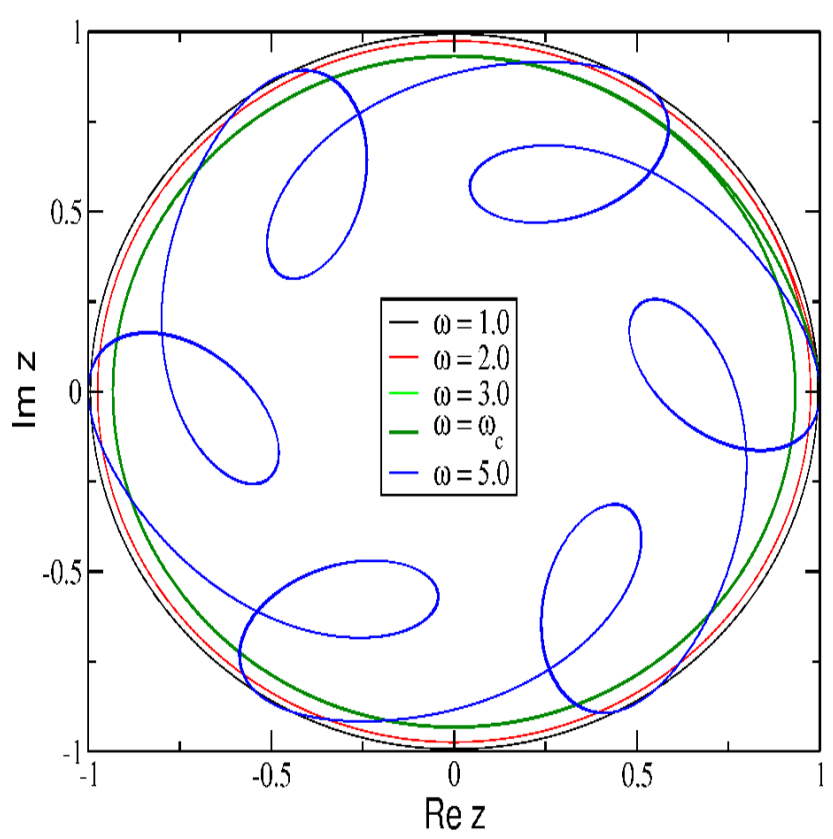

Fig. 4. Curves described in the complex plane by the order parameter (15) for a star of degree $q=3$ and different values of the pacemaker natural frequency. That corresponding to the critical value is analytically computed while the others are obtained by numerical simulations. of a Cayley tree of arbitrary radius $R$ (see Fig. 6). There are two main considerations for the general case. First, we notice that the time evolution of the effective frequency of the pacemaker and its

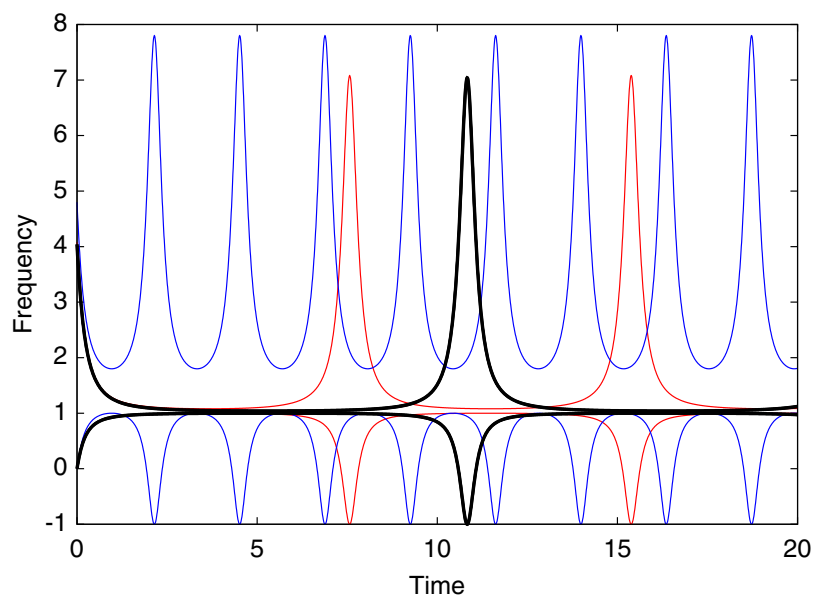

Fig. 5. Time evolution of the effective frequencies of the pacemaker (up) and its neighbors (down) in star of coordination number $q=3$ for different values of the pacemaker natural frequency $\omega>\omega_{c}: \omega=1.01 \omega_{c}$ (black line), $\omega=1.05 \omega_{c}$ (red line), $\omega=1.20 \omega_{c}$ (blue line). 


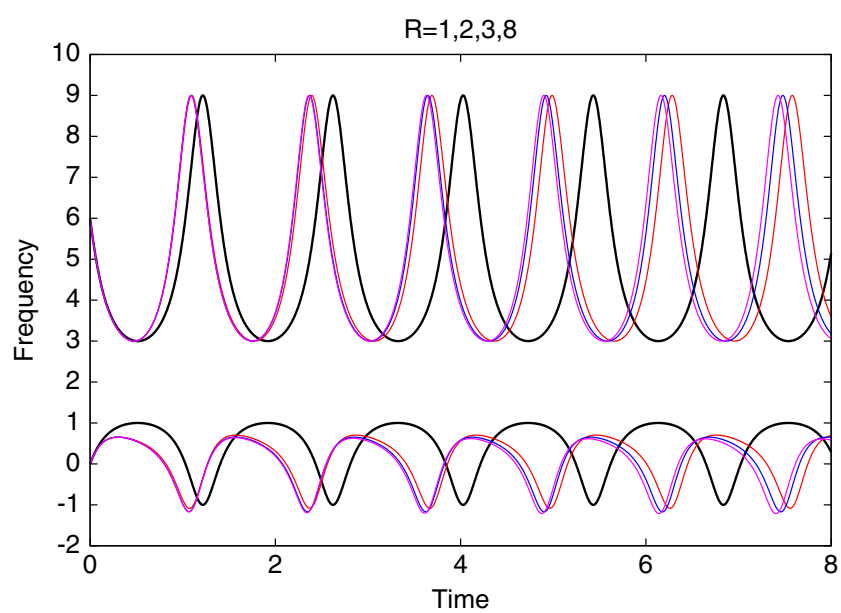

Fig. 6. Comparison of the time evolution of the effective frequencies of the pacemaker (up) and its neighbors (down) in Cayley trees of coordination number $q=3$ and different radii. The radii are respectively $R=1$ (black line, analytically computed), $R=2$ (red line), $R=3$ (blue line) and $R=8$ (pink line).

neighbors, changing the radius $R$ (and consequently the size $N$ ), given the same $\omega$, differ from the corresponding quantities for a star of equal $q$, just for the oscillation period. Actually, they share the same maximum and minimum values, and it is possible to transform one curve into another rescaling time $t \rightarrow t^{\prime}=\alpha t$. However, there is no trivial expression for the rescaling factor $\alpha$. Then we observe that, at least for large enough $\omega$, the perturbation introduced by the pacemaker on the dynamics in the more external shells can be neglected (see Fig. 7).
The frequencies of these units oscillate with a very small amplitude, when compared with those of the pacemaker and the most internal shell, around a mean value that is the same for all the oscillators that are not the leading one.

In terms of the order parameter this means that if we consider a Cayley tree with increasing radius, the last term in Eq. (3) becomes clearly dominant, and eventually, the perturbation introduced by the pacemaker will not be detected (see Fig. 7, right panel).

\section{Conclusions}

Analytical results are quite scarce when dealing with dynamical properties of nonlinear systems embedded in complex topologies. We have presented here a paradigmatic model with some assumptions that relax topological complexity, but nevertheless maintain the inherent dynamical complexity. Cayley trees or Bethe lattices have been the subject of many analysis in the literature because its recurrent topology facilitates the achievement of exact results. In our case, taking a Cayley tree as a reference structure, we have computed analytically the effect of a single pacemaker located at the root of the tree for a system of Kuramoto oscillators. We have then found exact values for the critical frequency of the pacemaker above which the system is not able to synchronize and also exact values for the order parameter introduced originally by Kuramoto. These results concern the relation

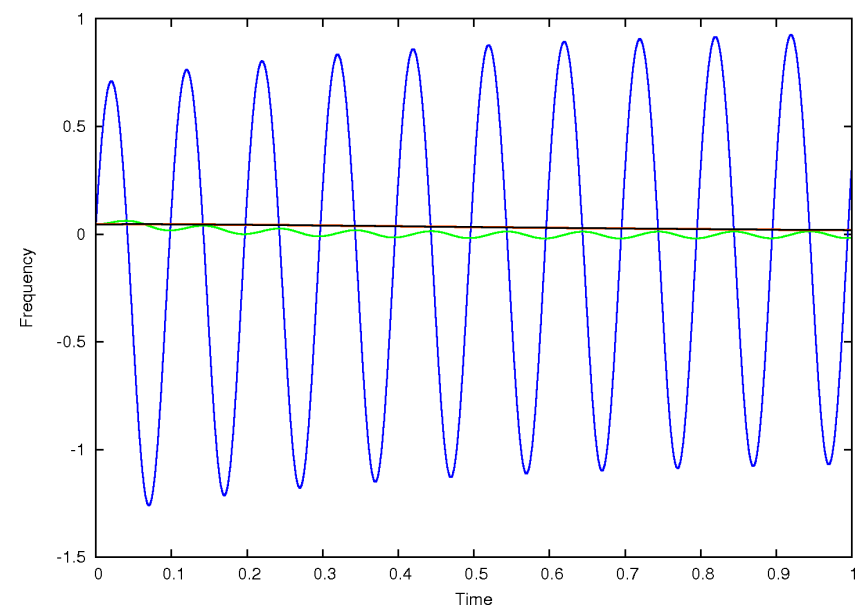

Fig. 7. Comparison of the effective frequencies time evolution in a Cayley tree of radius $R=4$ and coordination number $q=3$. The red line (left panel) corresponds to the pacemaker, the blue one to the nodes of the first shell, the others to the second (green) and the third one (black). In the right panel we have removed the pacemaker effective frequency zooming in on the others in order to show the hierarchical organization of the amplitudes of the frequency oscillation. The pacemaker natural frequency was 20 times larger than its critical value. 
between topology and dynamics in a system of interacting units in a tree structure. They provide important clues on this relation in a number of situations where the connectivity patterns are tree-like or have an almost tree-like topology, such as technological systems, like computer networks [Pastor-Satorras \& Vespignani, 2004], or biological systems, such as prokaryotic gene regulatory networks [Balazsi et al., 2005].

\section{Acknowledgments}

P. M. Gleiser acknowledges financial support from grants Sectyp UNCuyo 06/C298 and Conicet PIP2010 11220090100349. L. Prignano, C. J. PérezVicente and A. Diaz-Guilera acknowledge support from Spanish MCINN (FIS2009-13730) and Generalitat de Catalunya (2009SGR00838).

\section{References}

Acebrón, J. A., Bonilla, L. L., Pérez-Vicente, C. J., Ritort, F. \& Spigler, R. [2005] "The Kuramoto model: A simple paradigm for synchronization phenomena," Rev. Mod. Phys. 77, 137-185.

Arenas, A., Díaz-Guilera, A., Kurths, J., Moreno, Y. \& Zhou, C. [2008] "Synchronization in complex networks," Phys. Rep. 469, 93-153.

Balazsi, G., Barabasi, A.-L. \& Oltvai, Z. N. [2005] "Topological units of environmental signal processing in the transcriptional regulatory network of escherichia coli," Proc. Natl. Acad. Sci. USA 102, 7841-7846.

Buzna, L., Lozano, S. \& Diaz-Guilera, A. [2009] "Synchronization in symmetric bipolar population networks," Phys. Rev. E 80, 066120.
Diaz-Guilera, A. \& Arenas, A. [2008] "Phase patterns of coupled oscillators with application to wireless communication," Lect. Not. Comp. Sci. 5151, 184-191.

Kori, H. \& Mikhailov, A. S. [2004] "Entrainment of randomly coupled oscillator networks by a pacemaker," Phys. Rev. Lett. 93, 254101.

Kuramoto, Y. [1975] "Self-entrainment of a population of coupled nonlinear oscillators," Int. Symp. Mathematical Problems in Theoretical Physics, Lecture Notes in Physics, Vol. 39, ed. Araki, H. (Springer, NY, USA), pp. $420-422$.

Kuramoto, Y. [1984] Chemical Oscillations, Waves, and Turbulence (Springer-Verlag, NY, USA).

Osipov, G. V., Kurths, J. \& Zhou, C. [2007] Synchronization in Oscillatory Networks (Springer, Berlin, Germany).

Pastor-Satorras, R. \& Vespignani, A. [2004] Evolution and Structure of the Internet: A Statistical Physics Approach (Cambridge University Press, Cambridge, UK).

Pikovsky, A., Rosenblum, M. \& Kurths, J. [2001] Synchronization (Cambridge University Press, Cambridge, UK).

Prignano, L. \& Diaz-Guilera, A. [2012] "Extracting topological features from dynamical measures in networks of Kuramoto oscillators," Phys. Rev. E 85, 036112.

Radicchi, F. \& Meyer-Ortmanns, H. [2006] "Entrainment of coupled oscillators on regular networks by pacemakers," Phys. Rev. E 73, 036218.

Sendiña-Nadal, I., Buldu, J., Leyva, I. \& Boccaletti, S. [2008] "Phase locking induces scale-free topologies in networks of coupled oscillators," PLoS ONE 3, e2644.

Winfree, A. T. [1980] The Geometry of Biological Time (Springer-Verlag, Berlin, Germany). 